\title{
Briefing: Bringing clarity and new understanding of smart and modular integrated construction
}

Wei Pan PhD, MSc, BSc(Eng), PgCert, MCIOB, GMICE, MHKIE, FHEA Professor, Department of Civil Engineering, The University of Hong Kong, Pokfulam, Hong Kong (Orcid:0000-0002-2720-3073) (corresponding author:wpan@hku.hk)
Zhiqian Zhang MSc, BEng

PhD Researcher, Department of Civil Engineering, The University of Hong Kong, Pokfulam, Hong Kong

Yi Yang PhD, MSc, BSC

Post-doc Fellow, Department of Civil Engineering, The University of Hong Kong, Pokfulam, Hong Kong (Orcid:0000-0002-9418-5661)

Modular construction has been promoted worldwide, with well demonstrated benefits. Modular integrated construction (MiC) was recently established in Hong Kong with the focus on integrated solutions to address high-rise buildings in high-density cities for productivity and competitiveness. Nevertheless, insufficient and inconsistent understanding exists. The University of Hong Kong has recently launched A Glossary of Modular Integrated Construction, which defines and illustrates over 170 terms related to MiC, off-site construction and smart project delivery, aiming at synergised understanding. The Glossary brings clarity and new understanding of modular and smart construction to the industry and community. With a focus on future building development in high-rise highdensity contexts, the Glossary should become an important aid for achieving wider adoption of modular construction and smart technologies.

\section{Introduction}

Modular construction has been promoted worldwide with benefits well demonstrated. Modular integrated construction (MiC) was recently established in Hong Kong to focus particularly on integrated solutions for achieving smart project delivery (SPD) and sustainable construction for high-rise buildings in highdensity cities. Pan and Hon (2020: p. 65) defined MiC as 'a game-changing disruptively innovative approach to transforming fragmented site-based construction of buildings and facilities into integrated value-driven production and assembly of prefinished modules, which will realise enhanced quality, productivity, safety and sustainability'.

$\mathrm{MiC}$ has been demonstrated with substantial benefits over conventional construction - for example, improved economic efficiency, fortified environmental sustainability and enhanced social harmony (Pan et al., 2020a). Nevertheless, insufficient and inconsistent understanding exists in the construction industry and knowledge community, which hinders the successful and wide adoption of MiC (Pan et al., 2019). Therefore, in December 2020, the Centre for Innovation in Construction and Infrastructure Development of The University of Hong Kong launched $A$ Glossary of Modular Integrated Construction (Pan et al., 2020b), with the aim of bringing clarity and new understanding of the industry and community about modular and smart construction.

As $\mathrm{MiC}$ represents the most advanced among the many off-site construction (OSC) methods (Gibb, 1999) and is powered by smart technologies (Zhang et al., 2020), this Glossary defines and illustrates over 170 terms in relation to OSC, MiC and SPD, aiming for synergised understanding. This briefing paper aims to elaborate on the significance and contributions of the Glossary by presenting the three main categories of terms included (i.e. OSC, $\mathrm{MiC}$ and SPD) and highlighting the advancements of this Glossary over others in the literature.

\section{Category of terms}

\section{$2.1 \quad$ OSC-related terms}

OSC is defined as the manufacturing and pre-assembly of building components, elements or modules before installation into their final locations (Gibb, 1999). Based on the extent of off-site works, this Glossary categorises OSC technologies into three levels, ranging from materials, components and subassembly through prefabricated non-volumetric pre-assembly and volumetric pre-assembly, to prefabricated, prefinished and prefurnished modules, where MiC represents the most advanced form of OSC (Figure 1).

Furthermore, this Glossary covers wide-ranging terms that are similar to OSC, such as 'off-site production/fabrication/ manufacturing', 'prefabrication' and 'industrialised building' (Gibb and Pendlebury, 2006). The whole collection of OSCrelated terms serves as a knowledge base for consistent communication and sharing in the construction industry.

\subsection{MiC-specific terms}

$\mathrm{MiC}$ is powered by advanced structures and materials for the creation of higher and stronger buildings and by smart technologies for quality and efficient project delivery. The MiCspecific terms in this Glossary (Figure 2) include but are not limited to those frequently used in modular design (e.g. 


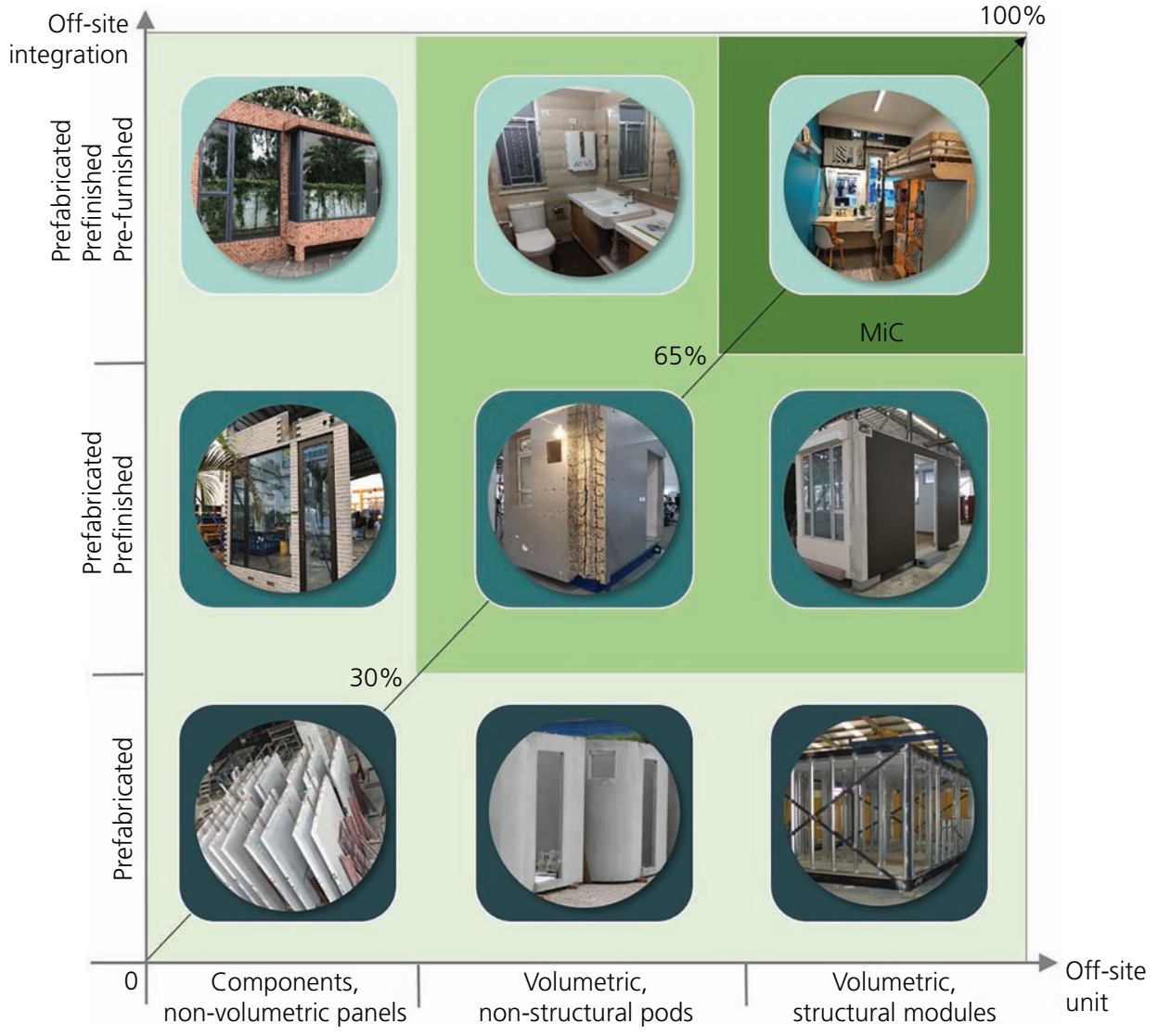

Figure 1. OSC: concept and categorisation

'modularisation'), project delivery process (e.g. 'concrete and steel-framed modules') and relevant regulations (e.g. 'practice note on gross floor area exemption').

Furthermore, this Glossary includes diverse terms related to modular construction in the wider context, such as 'prefabricated prefinished volumetric construction', established in Singapore, as well as 'volumetric modular construction', adopted in Australia. The comprehensive list of MiC-specific terms helps improve the common understanding of the modular approach and should facilitate relevant policymaking and project management in various jurisdictions.

\subsection{SPD-associated terms}

This Glossary covers a wide range of SPD-associated terms that have been well adapted to MiC/modular construction or have good potential to facilitate quality, safe and efficient project delivery. The selected terms cover wide-ranging technologies mainly residing in artificial intelligence (e.g. 'computer vision', 'deep learning'), Internet of things (IoT) (e.g. 'ultra-wideband sensors', 'cyber-physical system'), robotics (e.g. 'robot-oriented design', 'autonomous guided vehicle') and digital construction (e.g. 'building information modelling', 'virtual reality') (Figure 3).
The identified SPD-associated terms together reveal the co-evolution of smart building technologies and modular construction (Yang et al., 2019), indicating the clear trend of digital transformation of the whole industry. Thus, this Glossary should thus encourage the industry and research community to take active steps in exploring and implementing SPD technologies for successful MiC adoption.

\section{Discussion}

This Glossary contributes a comprehensive collection of terms in relation to OSC, MiC and SPD with cutting-edge prospects on industry development and practical generalisability to different settings. It should therefore advance other glossaries in the literature, which is discussed as follows.

First, this Glossary provides a collection of terms in a more comprehensive and systematic manner than similar glossaries do (e.g. MCCB, 2017). Besides those well-established terms (e.g. modular construction), this Glossary also includes a variety of emerging terms as relevant. For example, the term 'smart $\mathrm{MiC}^{\prime}$ ' is included to promote the implementation of digital, automatic and/ or intelligent technologies in the modular project delivery process to achieve high quality, safety, efficiency and sustainability. 


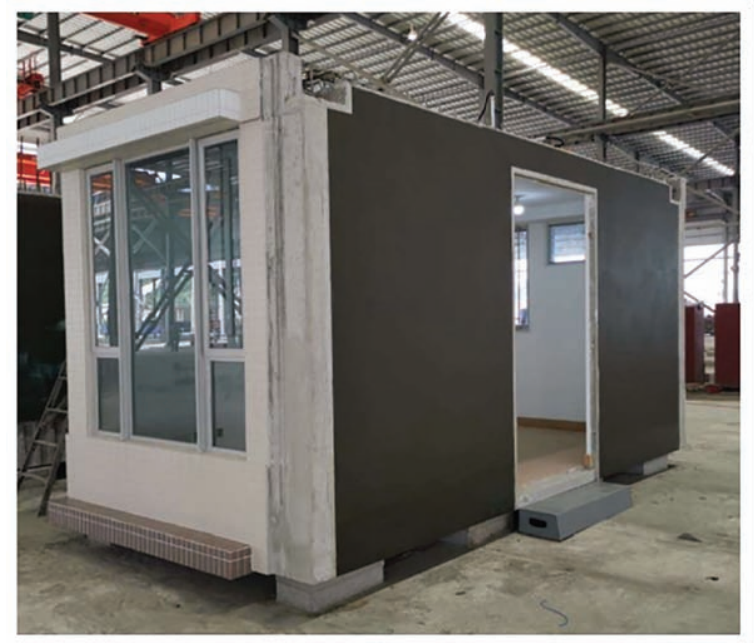

(a)

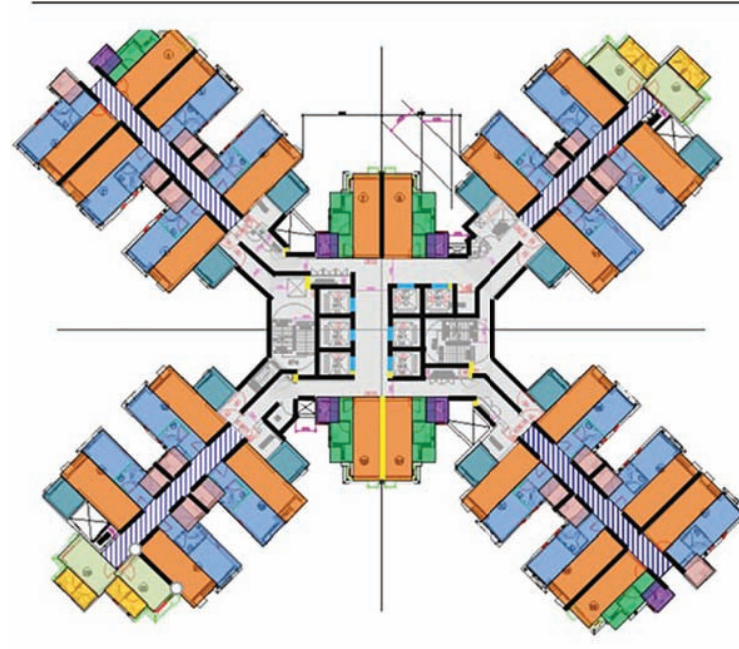

(c)

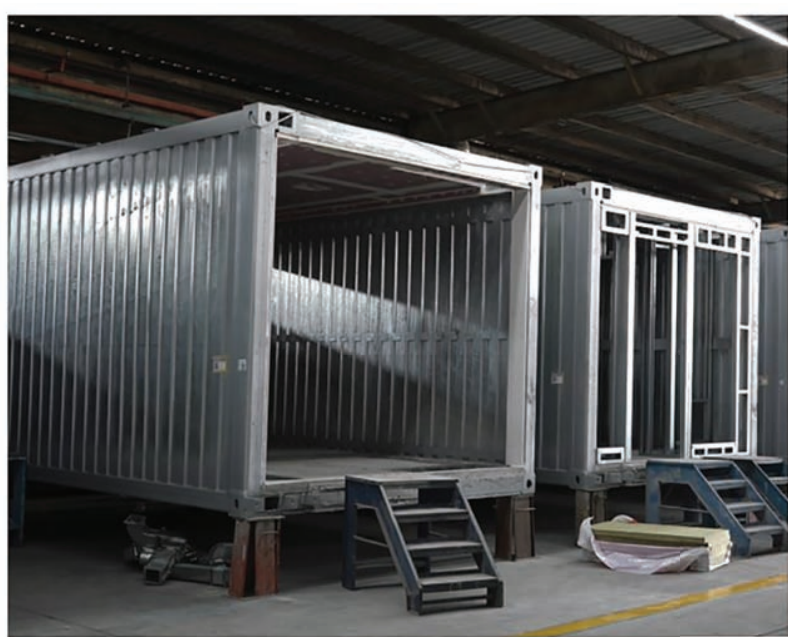

(b)

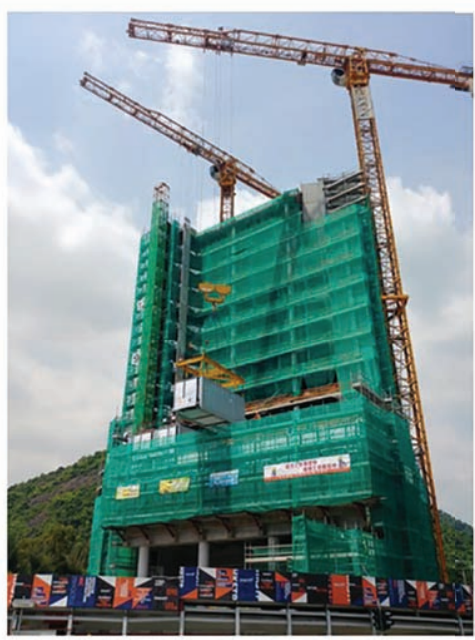

(d)

Figure 2. Examples of MiC-specific terms: (a) concrete module (courtesy of Yau Lee Wah); (b) steel-framed module (courtesy of China International Marine Containers (Group) Ltd); (c) modularisation; (d) module integrated construction (courtesy of Hip Hing)

Moreover, this Glossary presents a whole list of innovative technologies (e.g. terms in relation to $\mathrm{AI}$ and IoT) and their potential applications in MiC, which have never been sufficiently considered in previous glossaries.

Second, this Glossary presents cutting-edge prospects on the future research and development of the construction industry. For example, the term 'MiC higher' is included, which describes a future version of $\mathrm{MiC}$ involving the exploration of four interrelated themes in the framework of innovating higher: higher rise, higher density, higher tech and higher co-creation. Particularly, 'higher tech' promotes the integrated use of technologies such as AI, IoT, automation and robotics to transform digitally the traditional construction industry and thus further improve construction productivity, quality and sustainability, while 'higher co-creation' advocates industry-wide partnership for achieving 'higher tech'.

Third, this Glossary is generalisable and thus applicable for the construction industry of Hong Kong and other high-rise highdensity city contexts and beyond. Specifically, modular technologies used in different jurisdictions are depicted and compared. Furthermore, a number of representative research and development organisations worldwide are identified and provided. This information makes this Glossary an all-inclusive and useful source of information for the global construction industry and research community and supports the application and advancement of modular technologies. 


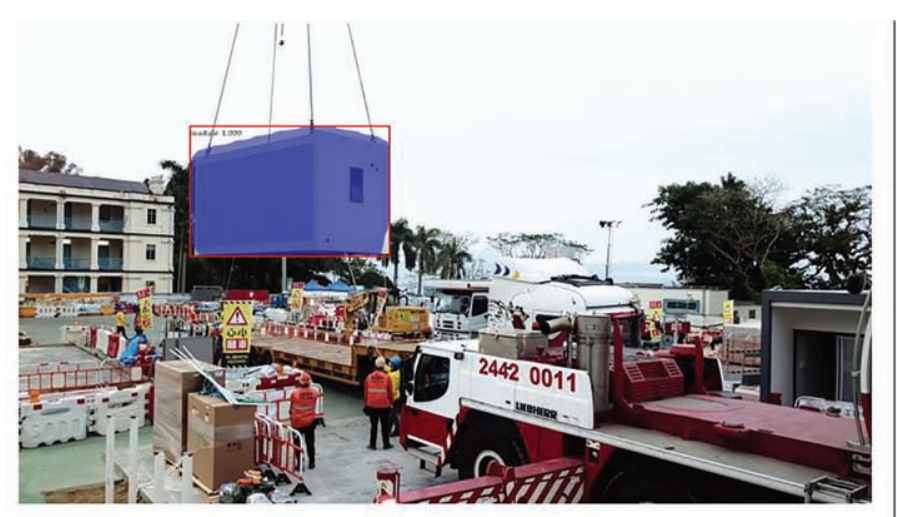

(a)

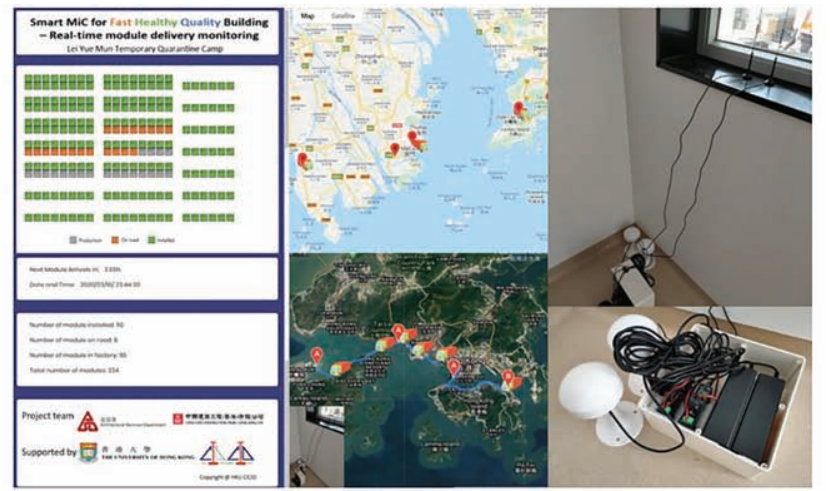

(b)

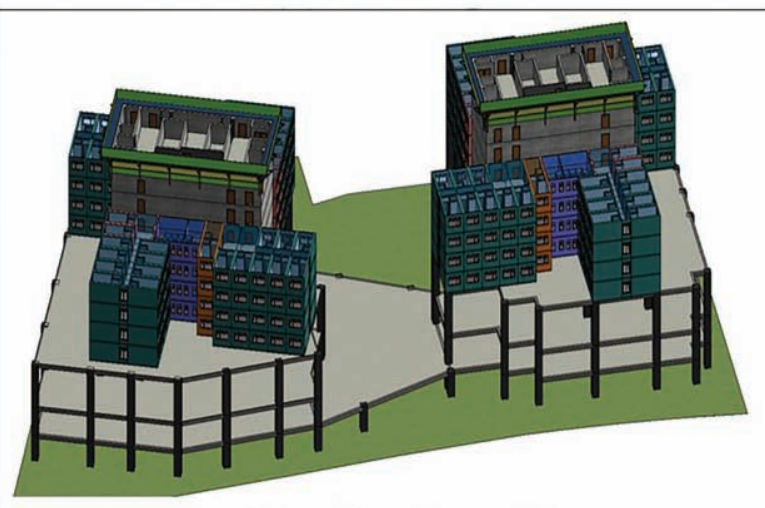

(d)

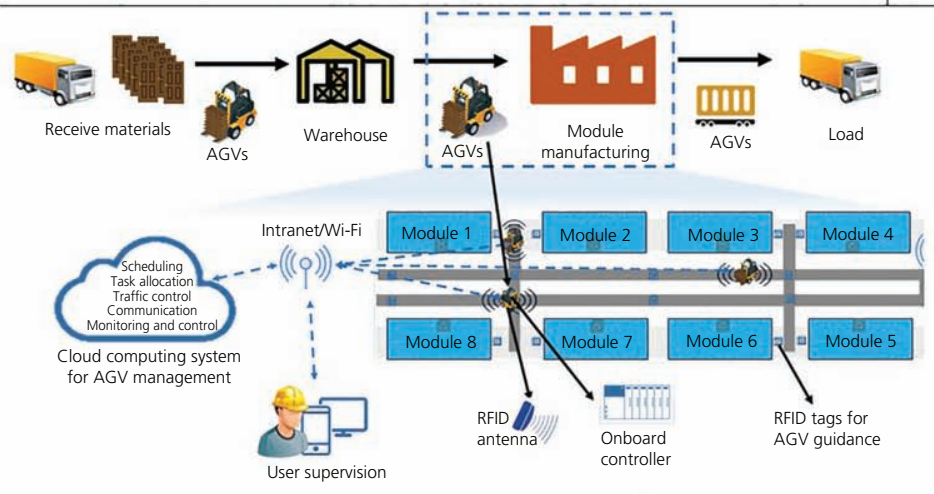

(c)

Figure 3. Examples of SPD-associated terms: (a) computer vision (Zhang et al., 2020); (b) Internet of things (Zhang et al., 2020); (c) autonomous guided vehicle (AGV) (Yang and Pan, 2020); (d) building information modelling

\section{Conclusions}

This briefing paper has introduced the purpose, contents and contributions of the booklet A Glossary of Modular Integrated Construction. This Glossary provides definitions of MiC in Hong Kong and related terms worldwide, accompanied by illustrations drawing on the state-of-the-art literature of modular and OSC and SPD. This Glossary should support construction stakeholders in their adoption of $\mathrm{MiC}$ by enhancing a comprehensive and consistent body of knowledge. The Glossary should also become an important aid in ensuring maximised benefit from adopting smart and modular construction and leapfrogging the construction industry to the future. By introducing the Glossary, this paper should assist both industry practitioners and academic researchers in quickly understanding the body of knowledge of $\mathrm{MiC}$ in a systematic manner.

\section{Acknowledgements}

We acknowledge support from the Research Impact Fund of the Hong Kong Research Grants Council (Project No.: HKU R702718) and the Strategic Public Policy Research Funding Scheme from the Policy Innovation and Co-ordination Office of the Government of the Hong Kong Special Administrative Region (Project No.: S2019.A8.013.19S).

\section{REFERENCES}

Gibb AGF (1999) Off-site Fabrication: Prefabrication, Preassembly, and Modularisation. Whittles Publishing, New York, NY, USA.

Gibb A and Pendlebury M (2006) Glossary of Terms. Buildoffsite and Loughborough University, Loughborough, UK.

MCCB (Modular Construction Codes Board) (2017) Handbook for the

Design of Modular Structures. MCCB, Monash University, Melbourne, Australia.

Pan W and Hon CK (2020) Briefing: Modular integrated construction for high-rise buildings. Proceedings of the Institution of Civil Engineers Municipal Engineer 173(2): 64-68, https://doi.org/10.1680/jmuen.18. 00028.

Pan W, Yang Y, Zhang Z and Chan S (2019) Modularisation for Modernisation: A Strategy Paper Rethinking Hong Kong Construction. The University of Hong Kong, Hong Kong. See https:// www.miclab.hk/mfm (accessed 20/03/2021).

Pan W, Zhang Z, Xie M and Ping T (2020a) Modular Integrated Construction for High-rises: Measured Success. The University of Hong Kong, Hong Kong. See https://www.miclab.hk/success (accessed 20/03/2021).

Pan W, Zhang Z and Yang Y (2020b) A Glossary of Modular Integrated Construction. The University of Hong Kong, Hong Kong. See http:// hdl.handle.net/10722/297012 (accessed 20/03/2021) and https://www. miclab.hk/glossary (accessed 20/03/2021).

Yang Y and Pan W (2020) Automated guided vehicles in modular integrated construction: potentials and future directions. Construction Innovation: Information, Process, Management 21(1): 85-104, https:// doi.org/10.1108/CI-07-2019-0073. 
Smart Infrastructure and Construction Volume 173 Issue 1
Briefing: Bringing clarity and new understanding of smart and modular integrated construction

Pan, Zhang and Yang
Yang Yi, Pan M and Pan W (2019) 'Co-evolution through interaction' of innovative building technologies: the case of modular integrated construction and robotics. Automation in Construction 107: article 102932, https://doi.org/10.1016/j.autcon.2019.102932.
Zhang Z, Pan W and Zheng Z (2020) Fighting Covid-19 through fast delivery of a modular quarantine camp with smart construction. Proceedings of the Institution of Civil Engineers - Civil Engineering, https://doi.org/10.1680/jcien.20.00025.

\section{How can you contribute?}

To discuss this paper, please email up to 500 words to the editor at journals@ice.org.uk. Your contribution will be forwarded to the author(s) for a reply and, if considered appropriate by the editorial board, it will be published as discussion in a future issue of the journal.

Proceedings journals rely entirely on contributions from the civil engineering profession (and allied disciplines). Information about how to submit your paper online is available at www.icevirtuallibrary.com/page/authors, where you will also find detailed author guidelines. 